\title{
STOCHASTIC GAMES, AN OVERVIEW
}

\section{O.J. Vrieze, Rijksuniversiteit Limburg, NL}

Stochastic games can be viewed as an integration of two other classes of mathematical decision problems, namely Noncooperative Games and Markovian Decision Problems. With Noncooperative Games Stochastic Games have the same aspect in common, which concerns the decision situation in which two or more decision makers (players) have to take a decision and where these decision mutually influence each others outcome. With Markovian decision problems Stochastic Games have in common the aspect that the decision process proceeds dynamically along a stochastic process based on Markov chains.

In stochastic games usually the infinite horizon case is considered, in which the set of discrete decision moments coincides with the set of natural numbers.

The theory of Stochastic Games started in 1953 with an initiating paper of Shapley. The theory has been developed into different branches. We mention: two-person zero-sum models (one player has to pay the other), nonzero-sum models (each player has his own reward-function), discounted models (future rewards are discounted in order to intercompare them), limiting-average models (the overall evaluation of a stream of rewards is taken as some average).

The theory of zero-sum models is more or less completed. In 1980 Mertens and Neyman showed that the last open question, namely the existence of the value, can be answered affirmatively. For nonzero-sum models still many questions are open. The main problems arise from the fact that, unlike in the zero-sum case, the limiting average models cannot be treated as limits of discounted models. During this talk an overview of Stochastic Games will be presented. Specials emphasis will be given to evaluation rules like discounted criterium, limiting average criterium, total reward criterium and weighted reward criterium and their interrelation. For the nonzero-sum models the main difficulties will be explained with respect to existence proofs of equilibria. 